\title{
Dissipative Controller Design for Networked Control Systems via the Markovian Jump System Approach
}

\author{
Asep Najmurrokhman ${ }^{1,2}$, Bambang Riyanto $^{1}$, Arief Syaichu-Rohman ${ }^{1} \&$ \\ Hendrawan $^{1}$ \\ ${ }^{1}$ School of Electrical Engineering and Informatics, Bandung Institute of Technology, \\ Jalan Ganesa No.10 Bandung 40132, Indonesia \\ ${ }^{2}$ Dept. of Electrical Engineering, University of Jenderal Achmad Yani, \\ Jalan Terusan Jendral Sudirman, Cimahi 40533, Indonesia \\ Email: asepnajmu@yahoo.com
}

\begin{abstract}
This paper describes a dissipative controller design for networked control systems modeled by the Markovian jump system. Dissipativity refers to the existence of a supply rate dealing with the system such that the closed-loop system has a dissipative property. The main result of this paper is a solvability condition in terms of linear matrix inequalities for achieving dissipativity of a dynamic output feedback problem in networked control systems via the Markovian jump system approach. A numerical example is given to show the efficacy of the proposed design.
\end{abstract}

Keywords: dissipative controller; linear matrix inequalities; Markovian jump systems; networked control systems; output feedback.

\section{$1 \quad$ Introduction}

The tremendous advancement in communication technology and computer networks and the need for real-time requirements in the control process has caused the emergence of a new paradigm in control analysis and design, called 'networked control system' (NCS) [1]. An NCS is a control system in which the control loop is closed via a shared communication network [2]. A typical NCS is depicted in Figure 1 [3]. In this figure, $G$ is the generalized plant, $K$ is the controller to be designed, $w$ is the disturbance, $u$ is the control input that is generated by the controller, $y$ is the measurement, and $z$ is the controlled output.

There are some parameters that arise when using a network in an NCS, such as packet dropout, time delay, and limited bandwidth. These network parameters modify the external signal $(u, y)$ of the plant and the controller. The control input $\hat{u}$ going to the plant is no longer equal to the output of the controller, while the measured output of plant $y$ is not exactly known by the controller. It is well known that the presence of these network parameters can degrade the performance of the control loop significantly and lead to instability [4].

Received December $22^{\text {nd }}, 2011$, Revised December $6^{\text {th }}, 2012$, Accepted for publication December $29^{\text {th }}, 2012$. Copyright (C) 2013 Published by ITB Journal Publisher, ISSN: 2337-5779, DOI: 10.5614/j.eng.technol.sci.2013.45.1.3 


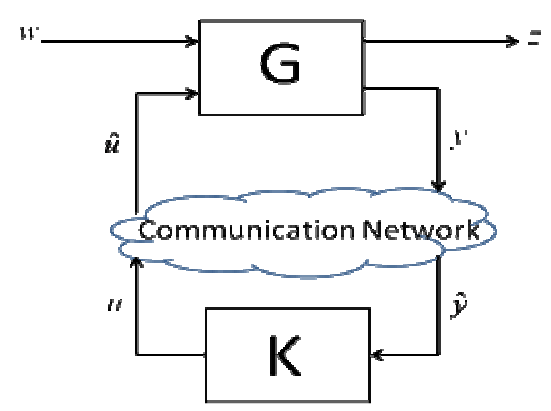

Figure 1 Block diagram of NCS.

Heemels, et al. [4] summarized that the network-induced imperfection constraints can be categorized into five types, as follows:

(a) Quantization errors in the signals transmitted over the network due to the finite word length of the packets

(b) Packet dropouts caused by the unreliability of the network

(c) Variable sampling/transmission intervals

(d) Varying communication delays

(e) Communication constraints caused by the sharing of the network by multiple nodes and the fact that only one node is allowed to transmit its packet per transmission.

Various methodologies have been proposed for modeling, stability analysis and controller designs for NCSs in the presence of these parameters. Hespanha, et al. [5] describe an NCS in tutorial fashion and include a direction of research to deal with them. Since the number of researches on NCSs is still growing, much of the available literature considers some of the above-mentioned types of network parameters while ignoring the other types. For example, several researchers describe the effect of communication delays, packet dropouts, and measurement quantization on NCS performance and derive a controller design that tackles these constraints [6-8]. Some authors address packet dropouts in NCS modeling and propose an exact characterization for controller synthesis [911]. Two papers consider the quantization effect in NCSs and the development of a controller to handle this effect in a closed-loop NCS $[12,13]$.

In controller design, existing methods can be classified into two main categories [8]. The first one is to design a controller first and then determine the network conditions, such as the maximum allowable transfer interval, to guarantee the stability and maintain a certain performance level, e.g. $[10,14]$. The second one is to explicitly incorporate the network-induced constraints using a certain model, e.g. the Markov process, into a controller design. The latter has recently 
emerged as the most popular approach, because the network-induced constraints are considered from the beginning of the analysis and synthesis of the NCS.

Meanwhile, the Markovian Jump System (MJS) has attracted increasing attention in recent literature, see e.g. [15-18] for linear systems, and [19] for nonlinear systems. Such systems have a transition between the models determined by a Markov chain. The MJS is considered to be appropriate to model plants whose structure is subject to random abrupt changes due to: component failure or repair, sudden environmental changes, abrupt variations of the operating point of a nonlinear plant, changing subsystem interconnections, and so on [9]. There is a quite deep literature for this class of systems [15]; previous studies that are relevant to this class of systems in NCS modeling were reported in $[8,9,16-21]$, only to name a few.

In this paper, we address the dissipative problem in designing a dynamic output feedback controller for NCSs via a MJS framework. The dissipative concept used to analyze and design the control system was initially developed by Willems [22]. This concept concerns the analysis and design of control systems that use input-output properties based on the energy-related description [23]. Practically speaking, a system has a dissipative property if it always dissipates energy. This property is an expected behavior, since the storage function of the underlying system is closely related to the system's energy and serves as a candidate for the Lyapunov function. When the dissipativity is assured, the stability problem can be solved. Meanwhile, the dissipative performance is a generalization of the performance measure, such as finite gain $\left(\mathrm{H}_{\infty}\right)$ and passivity [22]. Thus, the development of a dissipative framework will generalize the existing ones, including $\mathrm{H}_{\infty}$ control as well as passivity-based design.

Using a mathematical abstraction of the notions of physical power and energy, researchers have developed a stability analysis and designed a controller for various applications in dissipative system frameworks. Recent studies concerning the dissipative approach that are in line with our interest are reported in [24-28]. Aliyu [24] has proposed a dissipativity analysis for the nonlinear Markovian jump system. Zhang, et al. [25] have considered the dissipative problem of a class of stochastic hybrid systems and focused on the analysis of whether a stochastic hybrid system with time delay is stochastically asymptotically stable and strictly dissipative. Mahmoud, et al. [26] have worked on a robust dissipative control problem applied to a class of hybrid multi-rate control models with time-delay and a switching controller. Zhang, et al. [27] derived a linear state feedback controller in a reliable dissipative control problem for a class of stochastic hybrid systems. Wang, et al. [28] addressed the dissipative problem for uncertain time-delay NCSs. The aforementioned papers consider the effect of time delay in the system. So far, to the best of our 
knowledge, a dissipative control design for NCSs modeled by the MJS has not been investigated yet. The development of such a framework would generalize the existing ones, including $\mathrm{H}_{\infty}$ control and passivity-based NCS design.

Preliminary results of an NCS control design using the MJS have been published in [29]. The present paper extends the work in [29] by providing a complete derivation of the NCS design formula and presenting a numerical simulation to demonstrate its effectiveness.

For convenience, we adopt the following notations in this paper. Capital letters denote matrices and small letters denote vectors. For real matrices or vectors, (.) $)^{T}$ indicates their transpose. For a real square matrix $P$, we write $P>0$ (resp., $P$ $<0)$ if $P=P^{T}$ is symmetric and positive (resp. negative) definite. Matrices and vectors, if their dimensions are not explicitly stated, are assumed to be compatible for algebraic operations. The symbol (*) denotes each symmetric block of matrices. $\Re^{\mathrm{n}}$ denotes the space of all $n$-dimensional vectors. The symbol $\varepsilon\{X\}$ denotes the mathematical expectation of X. 0 denotes the zero scalar as well as zero matrices with appropriate dimensions. $\operatorname{Pr}(\mathrm{X})$ means the probability of a random variable $\mathrm{X}$. The set of integer numbers is denoted by $\boldsymbol{\aleph}$, i.e. $\boldsymbol{N}=\{1, \ldots, N\}$.

\section{Stability and Dissipativity Analysis of MJS}

Let's consider the following discrete-time systems

$$
\begin{aligned}
& x(k+1)=A(r(k)) x(k)+B(r(k)) w(k) \\
& z(k)=C(r(k)) x(k)+D(r(k)) w(k)
\end{aligned}
$$

where $x \in \mathfrak{R}^{n}$ is the state vector, and $w \in \mathfrak{R}^{p}$ and $z \in \mathfrak{R}^{q}$ are input and output respectively. The state matrices are functions of a discrete-time Markov chain taking values in a finite set $\boldsymbol{N}=\{1, \ldots, N\}$. The Markov chain has transition probabilities $p_{i j}=\operatorname{Pr}(r(k+1)=j \mid r(k)=i)$ which satisfy $p_{i j}>0$ and $\sum_{j=1}^{N} p_{i j}=1$ for each $i \in \mathfrak{W}$. The plant initial conditions are given by specifying $r(0)$ and $x(0)$. When the plant mode is in mode $i \in \mathfrak{N}$ (i.e. $r(k)=i$ ) in the sequel, we will use the following notation: $A_{i}:=A(r(k)=i), B_{i}:=B(r(k)=i)$, $C_{i}:=C(r(k)=i)$, and $D_{i}:=D(r(k)=i)$. Plants of this form are called discretetime MJS [21].

Concerning the stability analysis of MJS, there are several forms of stability that are summarized in Definition 1, as follows [21]. 
Definition 1. Given system (1) with $w(k)=0$, the equilibrium point at $x=0$ has the following properties:

(a) Mean square stable (MSS) if for every initial state $\left(x_{0}, r_{0}\right)$

$$
\lim _{k \rightarrow \infty} \mathcal{E}\left\{\left.x(k)^{T} x(k)\right|_{x_{0}, r_{0}}\right\}=0
$$

(b) Stochastically stable (SS) if for every initial state $\left(x_{0}, r_{0}\right)$

$$
\mathcal{E}\left\{\left.\sum_{k=0}^{\infty} x^{T}(k) x(k)\right|_{x_{0}, r_{0}}\right\}<\infty
$$

(c) Exponentially MSS (EMSS) if for every initial state $\left(x_{0}, r_{0}\right)$, there exists constants $0<\alpha<1$ and $\beta>0$ such that

$$
\varepsilon\left\{\left.x(k)^{T} x(k)\right|_{x_{0}, r_{0}}\right\}<\beta \alpha^{k} x_{0}^{T} x_{0}
$$

All such definitions are referred to in the literature as second-moment stability (SMS). In this paper, the notion of stability will be in the sense of SMS. Moreover, the method for checking the stability of MJS is given in the following proposition.

Proposition 1 [21]. System (1) with $w(k)=0$ is SMS if and only if there exist positive definite matrices $P_{i}$ that fulfill the following inequalities

$$
A_{i}^{T} P_{p i} A_{i}-P_{i}<0
$$

for all $i \in \boldsymbol{N} . P_{p i}$ are matrices produced from $P_{i}$ after multiplication by the transition probability of the associated Markov chain and given in the following form:

$$
P_{p i}=\sum_{j=1}^{N} p_{i j} P_{j}
$$

For linear deterministic systems, matrices $P_{p i}$ are equal to constant matrices $P$, so that the stability condition deals with the existence of matrix $P$ which satisfies the inequality $A^{T} P A-P<0$. The proposition states that SMS is equivalent to finding $N$ positive definite matrices $P_{i}$.

Meanwhile, the dissipativity of a system represents a condition in which the internal storage of the system never exceeds the storage supplied to the system. 
This is an important tool for stability analysis, as described in [22], or for designing a stabilizing controller, for example with passivity-based control as a special case of dissipativity [30]. The concept of dissipativity used in this paper can be consulted in [23]. In this paper, we deal with the class of systems that are dissipative with respect to a quadratic supply rate that depends on the input and the output of the system. To be precise, consider discrete-time systems as in (1). A stochastic quadratic supply rate has the form

$$
s(z, w)=\varepsilon\left\{\left(\begin{array}{c}
z \\
w
\end{array}\right)^{T}\left(\begin{array}{cc}
Q & S \\
S^{T} & R
\end{array}\right)\left(\begin{array}{c}
z \\
w
\end{array}\right)\right\}
$$

where the dimensions of matrices $Q, S$, and $R$ are determined by those of $z$ and $w$, and where $Q$ and $R$ are symmetric matrices, respectively. The dissipativity analysis can be stated as in definition 2 below, adopted from [24], for discretetime cases.

Definition 2. System (1) is said to be dissipative with respect to a quadratic supply rate (7) if there is a function $V(k, x(k), r(k))=x(k)^{T} P(r(k)) x(k)$ with $P(r(k))>0$ for all $r(k) \in \mathfrak{N}$ such that the following inequality is satisfied

$$
\varepsilon\{V(k+1, x(k+1), r(k+1))-V(k, x(k), r(k))\} \leq \varepsilon\{s(z(k), w(k))\}
$$

In the literature, function $V$, if any, is referred to as a storage function $[22,25]$. As is well known, the $\mathrm{H}_{\infty}$-control problem and passivity are special cases of dissipativity [26]. Both former performance measures are covered by selecting some appropriate matrices in the quadratic supply rate (7). The LMI condition stated in [18] to represent $\mathrm{H}_{\infty}$-control analysis can be covered by (7) via choosing $Q=-I, S=0$, and $R=\gamma I$, while passive systems are dissipative with respect to the supply rate (7) with $R$ and $Q$ are zero matrices, respectively, while $S$ is equal to the identity matrix. Without loss of generality, we can make the assumption that $\mathrm{Q} \leq 0$. The following proposition characterizes SMS and the dissipativity properties of discrete-time MJS (1).

Proposition 2. Consider the system given by (1). This system is SMS and is said to be dissipative with respect to a quadratic supply rate (7) if there exists a positive definite matrix $P(r(k))$ for all $r(k) \in \mathfrak{\aleph}$, which satisfies the following LMI 


$$
\left(\begin{array}{cc}
A_{i}^{T} P_{p i} A_{i}-P_{i}-C_{i}^{T} Q C_{i} & * \\
B_{i}^{T} P_{p i} A_{i}-\left(Q D_{i}+S\right)^{T} C_{i} & B_{i}^{T} P_{p i} B_{i}-\left(R+S^{T} D_{i}+D_{i}^{T} S+D_{i}^{T} Q D_{i}\right)
\end{array}\right)<0
$$

which

$$
P_{p i}=\sum_{j=1}^{N} p_{i j} P_{j}
$$

Proof. The above proposition can be proved by using definition 2, then plug (1), (7), and (10) in the inequality (8) to get (9). While SMS is assured by the fact that the $(1,1)$ block of LMI (9) satisfies inequality (5).

By Schur complement, the dissipativity condition represented by LMI (9) can be written in the $4 \times 4$ matrix block as follows

$$
\left(\begin{array}{cccc}
P_{i} & * & * & * \\
S C_{i} & R+S^{T} D_{i}+D_{i}^{T} S & * & * \\
P_{p i} A_{i} & P_{p i} B_{i} & P_{p i} & * \\
-Q C_{i} & -Q D_{i} & 0 & -Q
\end{array}\right)>0
$$

It is well known that the existence of a positive storage function that guarantees the stability of a dissipative system is assured by the zero-state detectability condition of the underlying system $[19,23]$. In this case, we assume in the rest of this paper that such a condition is also fulfilled.

\section{Dissipative Controller Design}

This part will include the main results of our study. Consider the discrete-time MJS as follows:

$$
\left\{\begin{array}{c}
x(k+1)=A_{i} x(k)+B_{1 i} u(k)+B_{2 i} w(k) \\
z(k)=C_{z i} x(k)+D_{z 1 i} u(k)+D_{z 2 i} w(k) \\
y(k)=C_{y i} x(k)+D_{y i} w(k)
\end{array}\right.
$$

where all vectors refer to Figure 1 and all matrices are of compatible dimensions. The full order controller associated with (12) to realize a dynamic output feedback control problem is represented as follows

$$
\left\{\begin{array}{c}
x_{c}(k+1)=K_{A i} x_{c}(k)+K_{B i} y(k) \\
u(k)=K_{C i} x_{c}(k)+K_{D i} y(k)
\end{array}\right.
$$


Where $x_{c}(k)$ denotes the controller states with the same dimension as plant states $x(k)$. Given the state space (12), the goal is to determine the controller matrices in such a way that we achieve a dissipativity condition of the closedloop systems. Connecting the controller (13) to the system (12), the closed-loop form is represented as follows

$$
\left\{\begin{array}{c}
\tilde{x}(k+1)=\tilde{A}_{i} \tilde{x}(k)+\tilde{B}_{i} w(k) \\
z(k)=\tilde{C}_{i} \tilde{x}(k)+\tilde{D}_{i} w(k)
\end{array}\right.
$$

where $\tilde{x}(k)$ represents the augmented state given by

$$
\tilde{x}(k)=\left(\begin{array}{c}
x(k) \\
x_{c}(k)
\end{array}\right)
$$

and the indicated matrices are

$$
\begin{aligned}
& \tilde{A}_{i}=\left(\begin{array}{cc}
A_{i}+B_{1 i} K_{D i} C_{y i} & B_{1 i} K_{C i} \\
K_{B i} C_{y i} & K_{A i}
\end{array}\right) \\
& \tilde{B}_{i}=\left(\begin{array}{c}
B_{2 i}+B_{1 i} K_{D i} D_{y i} \\
K_{B i} D_{y i}
\end{array}\right) \\
& \tilde{C}_{i}=\left(\begin{array}{ll}
C_{z i}+D_{z 1 i} K_{D i} C_{y i} & D_{z 1 i} K_{C i}
\end{array}\right) \\
& \tilde{D}_{i}=D_{z 2 i}+D_{z 1 i} K_{D i} D_{y i}
\end{aligned}
$$

Dissipativity of the closed-loop form is derived by involving the stochastic quadratic supply rate (7) and apply LMI condition (11) to closed-loop equation (14). Based on Proposition 2, dissipativity is assured if there are some positive definite matrices $\tilde{P}_{i}$ for all $i \in \mathcal{\aleph}$ that satisfy inequality matrix

$$
\left(\begin{array}{cccc}
\tilde{P}_{i} & * & * & * \\
S \tilde{C}_{i} & R+S^{T} \tilde{D}_{i}+\tilde{D}_{i}^{T} S & * & * \\
\tilde{P}_{p i} \tilde{A}_{i} & \tilde{P}_{p i} \tilde{B}_{i} & \tilde{P}_{p i} & * \\
-Q \tilde{C}_{i} & -Q \tilde{D}_{i} & 0 & -Q
\end{array}\right)>0
$$

By employing the Schur complement to LMI (20), we can write that LMI as follows: 


$$
\Phi=\left(\begin{array}{cccc}
\tilde{P}_{i} & * & * & * \\
S^{\prime} \tilde{C}_{i} & R+S^{T} \tilde{D}_{i}+\tilde{D}_{i}^{T} S & * & * \\
\tilde{A}_{i} & \tilde{B}_{i} & \tilde{P}_{p i}^{-1} & * \\
-Q \tilde{C}_{i} & -Q \tilde{D}_{i} & 0 & -Q
\end{array}\right)>0
$$

From the previous explanation, it can be seen that the full order controller that imposes a closed-loop system has twice the order of the plant. Therefore, matrix inequality (21) needs auxiliary symmetric matrices $\tilde{P}_{i} \in \mathfrak{R}^{2 n \times 2 n}$ for all $i \in \mathfrak{K}$. Adopting a similar method as in [18], which we employed in our previous paper [29], we define a new form of $\tilde{P}_{i}$ as $2 n \times 2 n$ real matrices, partitioned as follows:

$$
\tilde{P}_{i}=\left(\begin{array}{cc}
\Xi_{i} & * \\
\Pi_{i}^{T} & \hat{\Xi}_{i}
\end{array}\right), \tilde{P}_{i}^{-1}=\left(\begin{array}{cc}
\Delta_{i} & * \\
\Lambda_{i}^{T} & \hat{\Delta}_{i}
\end{array}\right), \tilde{T}_{i}=\left(\begin{array}{cc}
\Delta_{i} & I \\
\Lambda_{i}^{T} & 0
\end{array}\right)
$$

where all blocks are $n \times n$ real matrices. It can be verified that

$$
\tilde{T}_{i}^{T} \tilde{P}_{i} \tilde{T}_{i}=\left(\begin{array}{cc}
\Delta_{i} & I \\
I & \Xi_{i}
\end{array}\right)
$$

A similar partition is also performed on matrices $\tilde{P}_{p i}$, as follows:

$$
\tilde{P}_{p i}=\sum_{j=1}^{N} p_{i j} \tilde{P}_{j}=\left(\begin{array}{cc}
\Xi_{p i} & * \\
\Pi_{p i}^{T} & \hat{\Xi}_{p i}
\end{array}\right)
$$

and denoting

$$
\tilde{P}_{p i}^{-1}=\left(\begin{array}{cc}
\Omega_{1 i} & * \\
\Omega_{2 i}^{T} & \Omega_{3 i}
\end{array}\right), \tilde{Q}_{i}=\left(\begin{array}{cc}
I & \Xi_{p i} \\
0 & \Pi_{p i}^{T}
\end{array}\right)
$$

it can be easily verified that

$$
\tilde{Q}_{i}^{T} \tilde{P}_{p i}^{-1} \tilde{Q}_{i}=\left(\begin{array}{cc}
R_{1 i} & I \\
I & \Xi_{p i}
\end{array}\right)
$$

Note that matrix inequality (23) and (26) are motivated by the matrix definiteness lemma stating that a matrix $P$ is positive definite if and only if there is a matrix $X$ such that it satisfies $X^{T} P X>0$. In the literature, $X$ is sometimes referred to as a congruence transformation of $P$. Thus, matrix definiteness is invariant under congruence transformation. As stated in [18], it is important to 
see that the four block matrices that define the inverse $\tilde{P}_{p i}^{-1}$ depend nonlinearly on the four block matrices $\tilde{P}_{p i}$. Since $\Omega_{1 i}^{-1}=\Xi_{p i}-\Pi_{p i} \hat{\Xi}_{p i}^{-1} \Pi_{p i}^{T}$, setting $\Pi_{i}$ such that $\Pi_{i}=-\hat{\Xi}_{i}$ the partitioned matrix in (26) becomes

$$
\tilde{Q}_{i}^{T} \tilde{P}_{p i}^{-1} \tilde{Q}_{i}=\left(\begin{array}{cc}
\left(\Xi_{p i}+\Pi_{p i}\right)^{-1} & I \\
I & \Xi_{p i}
\end{array}\right)
$$

Letting the particular choice of $\Pi_{i}=-\hat{\Xi}_{i}$ and constraining matrix $\Pi_{i}$ be symmetric and the negative definite matrix provide $\Pi_{i}=-\hat{\Xi}_{i}=\Delta_{i}^{-1}-\Xi_{i}$, which enables us to rewrite (27) in the form

$$
\tilde{Q}_{i}^{T} \tilde{P}_{p i}^{-1} \tilde{Q}_{i}=\left(\begin{array}{cc}
\Delta_{q i} & I \\
I & \Xi_{p i}
\end{array}\right)
$$

In the general case, without the particular choice $\Pi_{i}=-\hat{\Xi}_{i}$, the equality $\Omega_{1 i}=\Delta_{q i}$ does not hold any longer, but matrices $\Omega_{1 i}$ given by

$$
\Omega_{1 i}^{-1}=\Xi_{p i}-\Pi_{p i} \hat{\Xi}_{p i}^{-1} \Pi_{p i}^{T}
$$

satisfy the inequalities

$$
\Omega_{1 i}^{-1} \geq \sum_{j=1}^{N} p_{i j}\left(\Xi_{j}-\Pi_{j} \hat{\Xi}_{j}^{-1} \Pi_{j}^{T}\right) \geq \sum_{j=1}^{N} p_{i j} \Delta_{j}^{-1} \geq \Delta_{q i}^{-1}
$$

From the previous discussion, matrix inequality (21) holds if and only if there is a congruence transformation $\Psi$ such that it satisfies

$$
\Psi^{T} \Phi \Psi>0
$$

Pick a congruence transformation below

$$
\Psi=\operatorname{diag}\left(\tilde{T}_{i}, I, \tilde{Q}_{i}, I\right)
$$

inequality (31) will yield

$$
\left(\begin{array}{cccc}
\tilde{T}_{i}^{T} \tilde{P}_{i} \tilde{T}_{i} & * & * & * \\
S^{T} \tilde{C}_{i} \tilde{T}_{i} & R+S^{T} \tilde{D}_{i}+\tilde{D}_{i} S & * & * \\
\tilde{Q}_{i}^{T} \tilde{A}_{i} \tilde{T}_{i} & \tilde{Q}_{i}^{T} \tilde{B}_{i} & \tilde{Q}_{i}^{T} \tilde{P}_{p i}^{-1} \tilde{Q}_{i} & * \\
-Q \tilde{C}_{i} \tilde{T}_{i} & -Q \tilde{D}_{i}^{T} & 0 & -Q
\end{array}\right)>0
$$


Solve each block of matrix inequalities (33), apply (23), (30), and introduce some variables as follows:

$$
\begin{aligned}
& K_{i}=K_{D i} \\
& L_{i}=K_{C i} V_{i}^{T}+K_{D i} C_{y i} \Delta_{i} \\
& F_{i}=\Pi_{p i} K_{B i}+\Xi_{p i} B_{i} K_{D i} \\
& M_{i}=\Xi_{p i} A_{i} \Delta_{i}+\left(\Pi_{p i} K_{A i}+\Xi_{p i} B_{i} K_{C i}\right) \Lambda_{i}^{T}+\left(\Pi_{p i} K_{B i}+\Xi_{p i} B_{i} K_{D i}\right) C_{y i} \Delta_{i}
\end{aligned}
$$

then the solvability condition is summarized in the next theorem in order to render the dissipativity of the closed-loop form by dynamic output feedback.

Theorem 1. There exists a dynamic output feedback controller of the form (13) in order to render the dissipativity of closed loop-form (14) with respect to the quadratic supply rate (7) if there exist symmetric matrices $\Xi_{i}, \Delta_{i}, Z_{i j}$ and matrices $M_{i}, L_{i}, F_{i}, K_{i}, H_{i}$ of compatible dimensions satisfying the LMIs

$$
\left(\begin{array}{cccccc}
\Delta_{i} & * & * & * & * & * \\
I & \Xi_{i} & * & * & * & * \\
\alpha^{T} & \beta^{T} & \phi & * & * & * \\
\delta^{T} & \mu^{T} & \eta^{T} & H_{i}+H_{i}^{T}-Z_{p i} & * & * \\
M_{i} & \kappa^{T} & \lambda^{T} & I & \Xi_{p i} & * \\
\pi^{T} & \sigma^{T} & \rho^{T} & 0 & 0 & -Q
\end{array}\right)>0
$$

and

$$
\left(\begin{array}{cc}
Z_{i j} & H_{i}^{T} \\
* & \Delta_{j}
\end{array}\right)>0
$$

for all $i, j \in \mathbb{N}$. Extra matrix variables in the LMI (38) are as follows

$$
\begin{array}{lc}
\alpha=\left(\Delta_{i}^{T} C_{z i}^{T}+L_{i}^{T} D_{z 1 i}^{T}\right) S & \beta=\left(C_{z i}^{T}+C_{y i}^{T} K_{i}^{T} D_{z 1 i}^{T}\right) S \\
\phi=R+S^{T}\left(D_{z 2 i}+D_{z 1 i} K_{i} D_{y i}\right)+\left(D_{z 2 i}+D_{z 1 i} K_{i} D_{y i}\right)^{T} S \\
\delta=\Delta_{i}^{T} A_{i}^{T}+L_{i}^{T} B_{1 i}^{T} & \mu=A_{i}^{T}+C_{y i}^{T} K_{i} B_{1 i}^{T} \\
\eta=B_{2 i}^{T}+D_{y i}^{T} K_{i}^{T} B_{1 i}^{T} & \kappa=A_{i}^{T} \Xi_{p i}+C_{y i}^{T} F_{i}^{T} \\
\lambda=B_{2 i}^{T} \Xi_{p i}+D_{y i}^{T} F_{i}^{T} & \pi=-\left(\Delta_{i}^{T} C_{z i}^{T}+L_{i}^{T} D_{z 1 i}^{T}\right) Q
\end{array}
$$




$$
\sigma=-\left(C_{z i}^{T}+C_{y i}^{T} K_{i}^{T} D_{z 1 i}^{T}\right) Q \quad \rho=-\left(D_{z 2 i}+D_{z 1 i} K_{i} D_{y i}\right) Q
$$

Furthermore, whenever (38)-(39) are solvable, a suitable controller is provided by

$$
\left(\begin{array}{ll}
K_{A i} & K_{B i} \\
K_{C i} & K_{D i}
\end{array}\right)=\left(\begin{array}{cc}
\Pi_{p i} & \Xi_{p i} B_{i} \\
0 & I
\end{array}\right)^{T}\left(\begin{array}{cc}
M_{i}-\Xi_{p i} A_{i} Y_{i} & F_{i} \\
L_{i} & K_{i}
\end{array}\right)\left(\begin{array}{cc}
\Lambda_{i}^{T} & 0 \\
C_{y i} \Delta_{i} & I
\end{array}\right)^{T}
$$

with $\Pi_{i}=\Delta_{i}^{-1}-\Xi_{i}$ and $\Lambda_{i}=\Delta_{i}$ for all $i \in \aleph$.

Proof. Assume that (21) holds. Partitioning $\tilde{P}_{i}$ as in (22), $\tilde{P}_{p i}^{-1}$ as in (25), and multiplying (21) to the right by (32) and to the left by its transpose, we obtain

$$
\left(\begin{array}{cccccc}
\Delta_{i} & * & * & * & * & * \\
I & \Xi_{i} & * & * & * & * \\
\alpha^{T} & \beta^{T} & \phi & * & * & * \\
\delta^{T} & \mu^{T} & \eta^{T} & \Omega_{1 i} & * & * \\
M_{i} & \kappa^{T} & \lambda^{T} & I & \Xi_{p i} & * \\
\pi^{T} & \sigma^{T} & \rho^{T} & 0 & 0 & -Q
\end{array}\right)>0
$$

By using inequality (30), for $H_{i}=\Delta_{q i}$ and $Z_{i j}=\Lambda_{q i} \Lambda_{j}^{-1} \Delta_{q i}+\chi I$ with $\chi>0$, (39) can be verified and we obtain

$$
H_{i}+H_{i}^{\prime}-Z_{p i}=\Delta_{q i}-\chi I \geq \Omega_{1 i}-\chi I
$$

By taking $\chi>0$ sufficiently small, inequality (41) implies that (38) holds.

Theorem 1 provides a quite general result regarding the solvability condition for a dynamic output feedback control problem in order to render the dissipativity of closed-loop systems under the Markovian jump system framework. The generality of this theorem is provided by noting that the $\mathrm{H}_{\infty}$-norm control and the passivity condition are obtained by choosing the appropriate supply rate function in the general results. Specifically, the $\mathrm{H}_{\infty}$-norm control such that its norm less than $\gamma$, as addressed in [18], is obtained by selecting $Q=-I, S=0$, and $R=\gamma I$, while passivity is recovered by choosing $Q=R=0$ and $S=I$. When the conditions of Theorem 1 are fulfilled, the computation of a controller that solves the dissipative control problem of a dynamic output feedback for the Markovian jump system framework can be carried out by the following procedure: 
1. Defining the set of matrix variables $\Xi_{i}, \Delta_{i}, Z_{i j}, M_{i}, L_{i}, F_{i}, K_{i}, H_{i}$ for all $i, j$ $\in \mathbb{N} \times$ as a solution of LMI (38)

2. Setting $\Pi_{i}=\Lambda_{i}^{-1}-\Xi_{i}, \Lambda_{i}=\Delta_{i}, \Pi_{p i}=\sum_{j=1}^{N} p_{i j} \Pi_{j}$, and $\Xi_{p i}=\sum_{j=1}^{N} p_{i j} \Xi_{j}$

3. Constructing of controller matrices by inserting all above matrices into matrix equation (40).

\section{$4 \quad$ Application to a Class of Networked Control Systems}

To show the effectiveness of our design, this part considers a numerical simulation regarding dissipative controller synthesis for a class of NCSs that has packet dropout during data transmission from the sensor-to-controller channel as well as from the controller-to-actuator channel. Figure 2 illustrates the NCS under consideration.

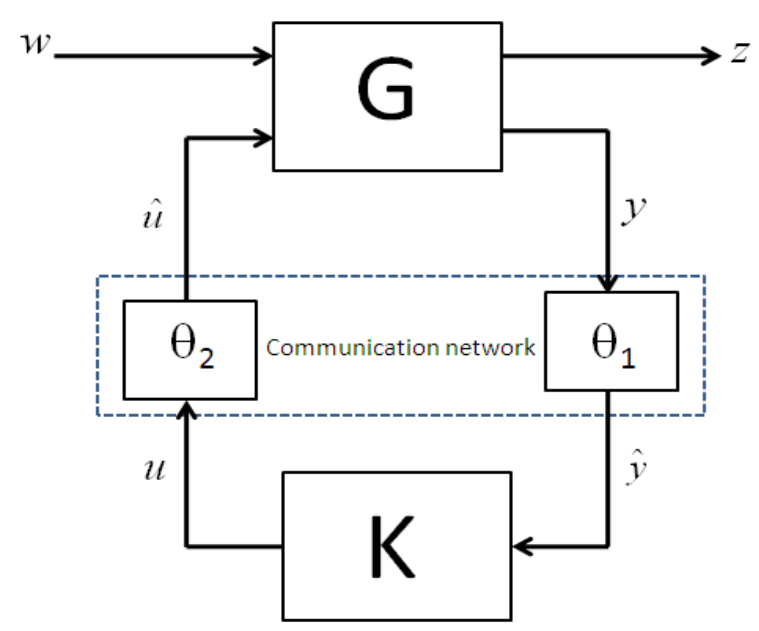

Figure 2 NCS with $\theta_{\text {i }}$ parameter.

In this figure, $\theta_{1}(k) \in\{0,1\}$ and $\theta_{2}(k) \in\{0,1\}$ denote the indicators of the packet dropouts in the sensor-to-controller and controller-to-actuator channels, respectively. A value of 0 indicates that the packet is dropped, while a value of 1 is used to denote the condition whenever the packet is transmitted successfully. Following the packet-dropout modeling by Seiler \& Sengupta [21], $\theta_{\mathrm{i}}$ is a Bernoulli process with the probability of dropout (i.e. $\theta_{\mathrm{i}}=0$ ) equal to $\alpha_{i} \in[0,1)$. For Bernoulli drops, the Markov chain only has two states and the transition probability from any state to the dropout state $\theta_{\mathrm{i}}=0$ is equal to $\alpha_{\mathrm{i}}$ and 
the transition probability from any state to the state $\theta_{\mathrm{i}}=1$ is equal to $1-\alpha_{\mathrm{i}}$, as shown in Figure 3.

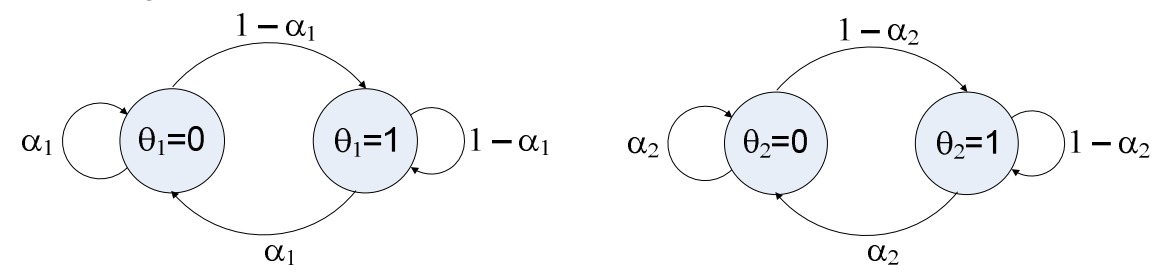

Figure 3 Two-state Markov chain for modeling packet dropout.

Define the set

$$
\Theta \equiv\left\{\left(\theta_{1}(k), \theta_{2}(k)\right): \theta_{1}(k) \in\{0,1\}, \theta_{2}(k) \in\{0,1\}\right\}
$$

Further define $r_{k} \in \mathfrak{\aleph} \equiv\{1,2,3,4\}$ and the one-to-one mapping $f: \Theta \rightarrow \mathfrak{\aleph}$ as follows:

$$
r_{k}=\left\{\begin{array}{l}
1,\left(\theta_{1}(k), \theta_{2}(k)\right)=(0,0) \\
2,\left(\theta_{1}(k), \theta_{2}(k)\right)=(0,1) \\
3,\left(\theta_{1}(k), \theta_{2}(k)\right)=(1,0) \\
4,\left(\theta_{1}(k), \theta_{2}(k)\right)=(1,1)
\end{array}\right.
$$

It is assumed that $r_{k}$ is driven by a discrete-time Markov chain and take values in with the transition probability $p_{i j}=\operatorname{Pr}\left(r_{k+1}=j \mid r_{k}=i\right)$ with $p_{i j} \geq 0$ and $\sum_{j} p_{i j}=1, \forall i, j \in \mathfrak{K}$. Since random variables of $\theta_{1}$ and $\theta_{2}$ are not correlated, it is clear that $p_{i 1}=\alpha_{1} \alpha_{2}, p_{i 2}=\alpha_{1}\left(1-\alpha_{2}\right), p_{i 3}=\alpha_{2}\left(1-\alpha_{1}\right)$, and $p_{i 4}=\left(1-\alpha_{1}\right)\left(1-\alpha_{2}\right)$. Furthermore, we consider the spring-mass-damper model taken from [31] as shown in Figure 4.

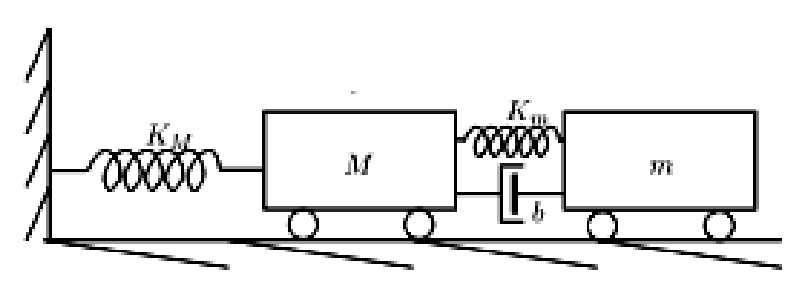

Figure 4 A spring-mass-damper model [31]. 
The first mass is attached to a fixed endpoint through another spring. The problem is to control the position and velocity of the second mass by applying a horizontal force on it. A state space representation in the form of continuous time of that model is taken from [18] as follows:

$$
\left\{\begin{array}{c}
\dot{x}=A x(t)+B_{1} u(t)+B_{2} w(t) \\
z(t)=C_{z} x(t)+D_{z 1} u(t)+D_{z 2} w(t) \\
y(t)=C_{y} x(t)+D_{y} w(t)
\end{array}\right.
$$

with each matrix is in the following:

$$
\begin{aligned}
& A=\left(\begin{array}{cccc}
0 & 0 & 1 & 0 \\
0 & 0 & 0 & 1 \\
-30.0 & 10.0 & -0.36 & 0.36 \\
5.0 & -5.0 & 0.18 & -0.18
\end{array}\right) \\
& B_{1}=\left(\begin{array}{llll}
0 & 0 & 0 & 1
\end{array}\right)^{T} \quad B_{2}=\left(\begin{array}{cccc}
0 & 1 & 0 & 0 \\
0 & 0 & 0 & 0
\end{array}\right)^{T} \\
& C_{z}=\left(\begin{array}{cccc}
0 & 50.0 & 0 & 0 \\
0 & 0 & 0 & 1.8 \\
0 & 0 & 0 & 0
\end{array}\right) \quad D_{z 1}=\left(\begin{array}{lll}
0 & 0 & 1
\end{array}\right)^{T} \quad D_{z 2}=\left(\begin{array}{ll}
0 & 0 \\
0 & 0 \\
0 & 0
\end{array}\right) \\
& C_{y}=\left(\begin{array}{cccc}
1 & 0 & 0 & 0 \\
0 & 0 & 1 & 0
\end{array}\right) \quad D_{y}=\left(\begin{array}{cc}
0 & 0.1 \\
0 & 0.125
\end{array}\right)
\end{aligned}
$$

In the context of NCS, the position and velocity of the first mass can be measured and delivered to the controller through the sensor-to-controller channel in the communication network. Subsequently, a controller is designed to overcome the packet dropout parameter which appears in the sensor-tocontroller and controller-to-actuator channels. By choosing a state in the Markov chain under consideration as in (44), the state space representation in the form of discrete-time (12) is as follows:

$$
\begin{aligned}
A_{i} & =\left(\begin{array}{cccc}
-0.7562 & 0.5086 & 0.07911 & 0.1435 \\
0.2092 & 0.5604 & 0.07175 & 0.4012 \\
-1.656 & 0.07364 & -0.7588 & 0.5113 \\
-0.1463 & -1.289 & 0.2556 & 0.514
\end{array}\right) \\
B_{12}=B_{14} & =\left(\begin{array}{lllll}
0.02304 & 0.111 & 0.1435 & 0.4012
\end{array}\right)^{T} \quad B_{11}=B_{13}=\left(\begin{array}{llll}
0 & 0 & 0 & 0
\end{array}\right)^{T}
\end{aligned}
$$




$$
\begin{aligned}
& B_{2 i}=\left(\begin{array}{cccc}
0.1252 & 0.4171 & 0.5086 & -0.4396 \\
0 & 0 & 0 & 0
\end{array}\right)^{T} \\
& C_{z i}=\left(\begin{array}{cccc}
0 & 50.0 & 0 & 0 \\
0 & 0 & 0 & 1.8 \\
0 & 0 & 0 & 0
\end{array}\right) \\
& D_{z 12}=D_{z 14}=\left(\begin{array}{lll}
0 & 0 & 1
\end{array}\right)^{T} \quad D_{z 11}=D_{z 13}=\left(\begin{array}{lll}
0 & 0 & 0
\end{array}\right)^{T} \\
& D_{z 2 i}=\left(\begin{array}{cc}
0 & 0 \\
0 & 0 \\
0 & 0
\end{array}\right) \\
& C_{y 2}=C_{y 4}=\left(\begin{array}{cccc}
1 & 0 & 0 & 0 \\
0 & 0 & 1 & 0
\end{array}\right) \quad C_{y 1}=C_{y 3}=\left(\begin{array}{cccc}
0 & 0 & 0 & 0 \\
0 & 0 & 0 & 0
\end{array}\right) \\
& D_{y 2}=D_{y 4}=\left(\begin{array}{cc}
0 & 0.1 \\
0 & 0.125
\end{array}\right) \quad D_{y 1}=D_{y 3}=\left(\begin{array}{ll}
0 & 0 \\
0 & 0
\end{array}\right)
\end{aligned}
$$

The discrete-time version of this model is obtained by discretization of the continuous model with a sampling time of $0.5 \mathrm{~s}$ and a zero order hold placed on each input. For the purpose of simulation, we choose $Q=-I, S=0$, and $R=\gamma I$ in the supply rate (7), which means that we deal with the $\mathrm{H}_{\infty}$-control performance as a special case of dissipative performance. Table 1 shows the performance of the system with different probabilities of packet dropout.

Table $1 \mathrm{H}_{\infty}$-norm of different probabilities of packet dropout.

\begin{tabular}{ccc}
\hline $\boldsymbol{\alpha}_{1}$ & $\boldsymbol{\alpha}_{\mathbf{2}}$ & \multicolumn{1}{c}{$\boldsymbol{\gamma}$} \\
\hline 0.1 & 0.1 & 32.5 \\
0.1 & 0.5 & 38.0 \\
0.5 & 0.5 & 56.9 \\
0.6 & 0.7 & 71.3 \\
0.7 & 0.8 & 75.4 \\
0.9 & 0.9 & 177.8 \\
\hline
\end{tabular}

From this table we know that the more packet dropout, the poorer the performance of the system. The mode transition during the simulation is depicted in Figure 5. Figures 6-8 shows the time-response of the controlled output and the control input of the closed-loop system under the specified mode transition as depicted in Figure 5. 


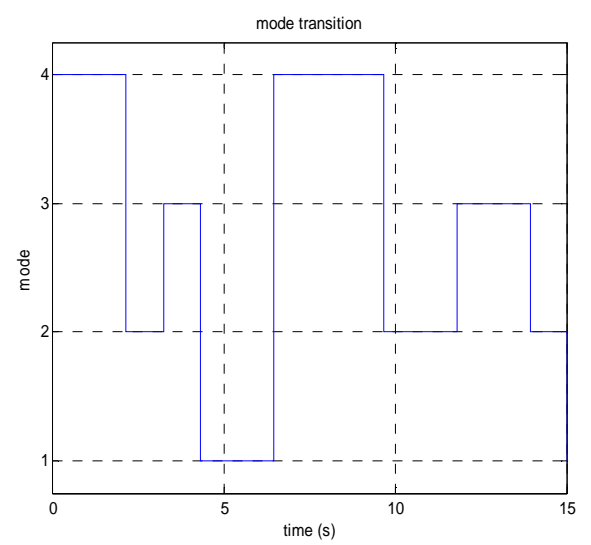

Figure 5 Mode transition.
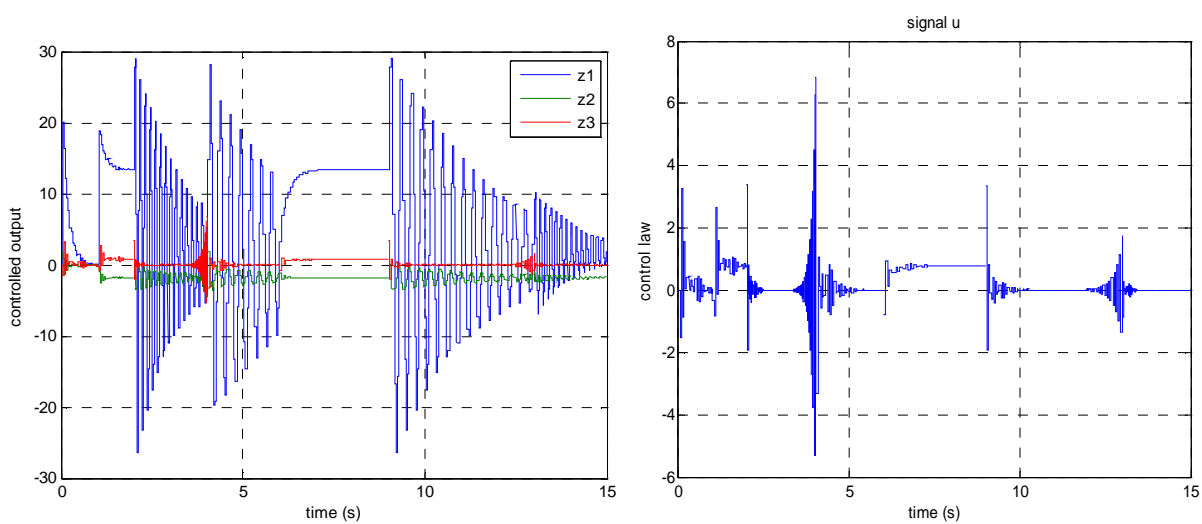

Figure 6 Time-response of controlled output and control input, respectively, for packet dropout equals $10 \%$.
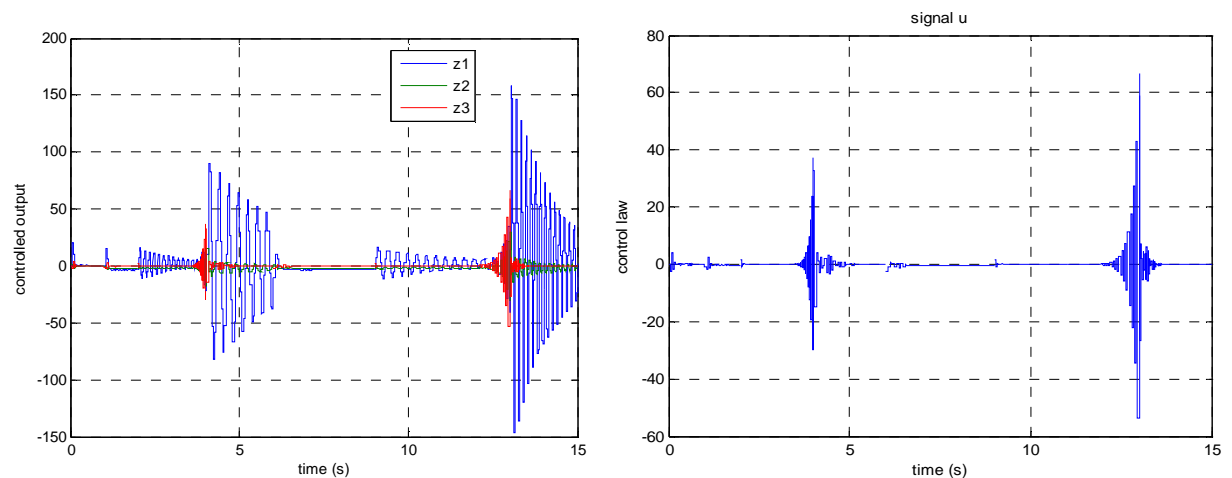

Figure 7 Time-response of controlled output and control input, respectively, when packet dropout equals $50 \%$. 



Figure 8 Time-response of controlled output and control input, respectively, when packet dropout equals $90 \%$.

It can be seen from all figures that the designed controller is capable of overcoming the packet dropout effect in the network. However, the simulation result deserves some remarks. We take the three values of probability of packet dropout, which indicate a good, medium, and bad condition of the network, respectively. First we consider the case when $\alpha_{i}=0.1$, which means the probability of successfully transmitting a data packet equals $90 \%$. As can be observed from Figure 6, the time response of the controlled output tends to converge with some spikes, which happen during inter mode transition. The amount of control input is small enough for a small probability of packet dropout. Next we take two other cases, which represent a medium and a bad condition of the network. As shown in Figures 7 and 8, the higher the probability of packet dropout, the more control input is required. The effect of the probability of packet dropout on the amount of control input is quite straightforward.

To test the robustness to modeling uncertainty, we employed a state matrix in the form of $A+\Delta A$ instead of $A$ in our simulation. The value of $\Delta A$ is given by the percentage amount to nominal plant $A$. By utilizing the algorithm proposed in the controller design, we obtained that an amount of $10 \%$ from the nominal plant is worst-case performance. Figure 9 and 10 show the time-response of the controlled output of the closed-loop system and the control input, respectively, with modeling uncertainty about $10 \%$ as the probability of packet dropout equals 0.1. From this simulation, we can observe that the proposed method renders the robustness of the closed-loop NCS to a prescribed modeling uncertainty. 


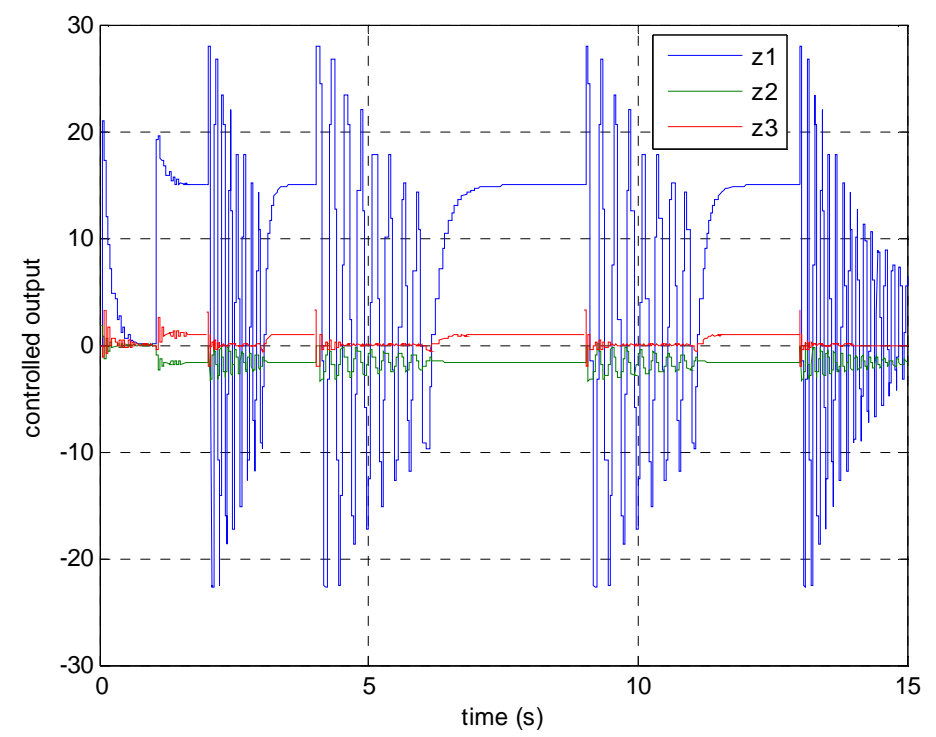

Figure 9 Time-response of controlled output with modeling uncertainty about $10 \%$ from nominal plant when packet dropout equals $10 \%$.

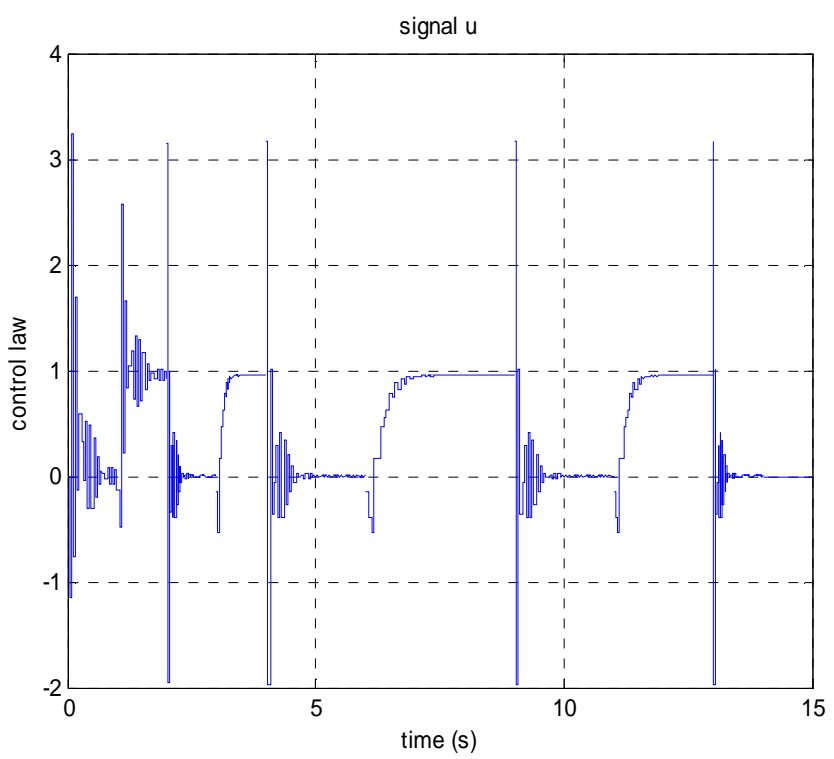

Figure 10 Time-response of control input with modeling uncertainty about $10 \%$ from nominal plant when packet dropout equals $10 \%$. 


\section{$5 \quad$ Conclusions}

This paper presents a dissipative controller design for a dynamic output feedback problem in NCSs modeled by the Markovian jump system. The main result is that the solvability condition of that problem, represented by two LMIs, was solved. Based on the solutions of the LMIs, we were able to construct a controller by plugging unknown variables in those LMIs into the controller matrices equation. We applied the results to a controller design for a class of NCSs that has packet dropout during data transmission from the sensor-tocontroller channel as well as from the controller-to-actuator channel. Our numerical simulation showed that the designed controller is capable of overcoming the packet dropout effect in the network, as well as rendering the robustness property to a prescribed modeling uncertainty. Further studies will be focused on the inclusion of a parametric uncertainty and then designing robust control systematically with dissipative performance, as well as exploring a nonlinear model of an NCS under the Markovian jump system framework.

\section{Acknowledgements}

We would like to thank Professor Romeo Ortega of Supelec, France, for our fruitful discussion concerning the dissipative concept during his visit of ITB. The first author is also indebted to André Fioravanti of INRIA, France, for providing his kind assistance on Matlab Code to solve a numerical example in his collaborative paper [18].

\section{References}

[1] Hokayem, P.F. \& Abdallah C.T., Inherent Issues in Networked Control Systems: A Survey, Proc. of the 2004 American Control Conference, pp. 4897-4902, June 30-July 2, 2004.

[2] Gupta, R.A. \& Chow, M.Y., Networked Control Systems: Overview and Research Trends, IEEE Trans. on Industrial Electronics, 57(7), pp. $2527-$ 2535, July 2010.

[3] Heemels, W.P.M.H. \& van de Wouw, N., Stability and Stabilization of Networked Control Systems, in Networked Control Systems, Bemporad, A., et al. (eds), Springer Verlag Berlin, pp. 203-253, 2010.

[4] Heemels, W.P.M.H, Teel, A.R., van de Wouw, N. \& Nesic, D., Networked Control Systems with Communication Constraints: Tradeoffs between Transmission Intervals, Delays and Performance, IEEE Trans. Automatic Control, 55(8), pp. 1781-1796, August 2010.

[5] Hespanha, J.P., Naghshtabrizi, P. \& Xu, Y., A Survey of Recent Results in Networked Control Systems, Proceedings of the IEEE, 95(1), pp. 138162, January 2007. 
[6] Lam, J., Gao, H. \& Wang, C., Stability Analysis for Continuous Systems with Two Additive Time Varying Delay Components, Systems and Control Letters, 56(1), pp. 16-24, January 2007.

[7] Gao, H., Chen, T. \& Lam, J., A New Delay System Approach to Networkbased Control, Automatica, 44(1), pp. 39-52, January 2008.

[8] Shi, Y. \& Yu, B., Output Feedback Stabilization of Networked Control Systems with Random Delays Modeled by Markov Chains, IEEE Trans. on Automatic Control, 54(7), pp. 1668-1674, July 2009.

[9] Aberkane, S., Ponsart, J.C. \& Sauter, D., Output-Feedback $\mathrm{H}_{2} / \mathrm{H}_{\infty}$ Control of A Class of Networked Fault Tolerant Control Systems, Asian Journal of Control , 10(1), pp. 34-44, January 2008.

[10] Hirche, S., Chen, C-C. \& Buss, M., Performance Oriented Control Over Networks: Switching Controllers and Switched Time Delay, Asian Journal of Control, 10(1), pp. 24-33, January 2008.

[11] Ishii, H., $H_{\infty}$ Control with Limited Communication and Message Loss, Systems and Control Letters, 57, pp. 322-331, 2008.

[12] Peng, C. \& Tian, Y-C., Network-based $H_{\infty}$ Control of Linear Systems with State Quantization, Journal of Information Sciences, 177(24), pp. 5763-5774, 2007.

[13] Xiao, N., Xie, L. \& Fu, M., Quantized Stabilization of Markov Jump Linear Systems via State Feedback, IEEE American Control Conf., pp. 4020-4025, June 10-12, 2009.

[14] Zhang, W., Branicky, M.S. \& Phillips, S.M., Stability of Networked Control Systems, IEEE Control Systems Magazine, 21(2), pp. 84-99, 2001.

[15] Costa, O.L.V., Fragoso, M.D. \& Marques, R.P., Discrete-Time Markov Jump Linear Systems, Springer-Verlag, London, UK, 2005.

[16] Li, L. \& Ugrinovskii, V.A., On Necessary and Sufficient Conditions for $H_{\infty}$ Output Feedback Control of Markov Jump Linear Systems, IEEE Trans. on Automatic Control, 52(7), pp. 1287-1292, July 2007.

[17] Fioravanti, A.R., Goncalves, A.P.C. \& Geromel, J.C., $H_{2}$ and $H_{\infty}$ Filtering of Discrete-time Markov Jump Linear Systems through Linear Matrix Inequalities, Proceedings of the 17th World Congress The International Federation of Automatic Control Seoul, Korea, pp. 26812688, July 6-11, 2008.

[18] Geromel, J. C., Goncalves, A.P.C. \& Fioravanti, A.R., Dynamic Output Feedback Control of Discrete-Time Markov Jump Linear Systems through Linear Matrix Inequalities, SIAM Journal on Control and Optimization, 48(2), pp. 573-593, 2009.

[19] Aliyu, M.D.S. \& Boukas, E.K., Robust H-infinity Control for Markovian Jump Nonlinear Systems, Journal of Mathematical Control and Information, 17, pp. 295-308, 2000. 
[20] Xiong, J. \& Lam, J., Robust $\mathrm{H}_{2}$ Control of Markovian Jump Systems with Uncertain Switching Probabilities, International Journal of Systems Science, 40(3), pp. 255-265, March 2009.

[21] Seiler, P. \& Sengupta, R., An $H_{\infty}$ Approach to Networked Control, IEEE Trans. on Automatic Control, 50(3), pp. 356-364, March 2005.

[22] Willems, J.C., Dissipative Dynamical Systems Part I: General Theory, Part II: Linear Systems with Quadratic Supply Rates, Arch. Rtion. Mech. Analysis, 45, pp. 321-393, 1972.

[23] Brogliato, B., Lozano, R., Maschke, B. \& Egeland, O., Dissipative Systems Analysis and Control, Springer-Verlag, London, 2007.

[24] Aliyu, M.D.S., Dissipativity and Stability of Nonlinear Jump Systems, Proc. of American Control Conference, pp. 795-799, June 1999.

[25] Zhang, H., Yan, H. \& Chen, Q., Stability and Dissipative Analysis for A Class of Stochastic System with Time-Delay, Journal of the Franklin Institute, 347, pp. 882-893, June 2010.

[26] Mahmoud, M.S., Nounou, H.N. \& Xia, Y., Robust Dissipative Control for Internet-Based Switching Systems, Journal of the Franklin Institute, 347(1), pp. 154-172, February 2010.

[27] Zhang, H., Guan, Z-H. \& Feng, G., Reliable Dissipative Control for Stochastic Impulsive Systems, Automatica, 44(2008), pp. 1004-1010, 2008.

[28] Wang, J., Han, Q.L. \& Yang, F., Robust Dissipative Control for Networked Control Systems with Multiple Packet Dropout, Proc. of the $18^{\text {th }}$ IFAC World Congress, pp. 78-83, August 28-Sept. 2, 2011.

[29] Najmurrokhman, A., Riyanto, B., Rohman, A.S. \& Ortega, R., Output Feedback Controller for Dissipative Networked Control Systems Via Markovian Jump System Approach, Proceedings of International Conference on Intelligent Unmanned Systems (ICIUS), Bali, Indonesia, pp. 510-515, Nov 3-5, 2010.

[30] El Hawwary, M.I., Passivity Methods for The Stabilization of Closed Sets in Nonlinear Control Systems, PhD Dissertation, Dept. of Electrical and Computer Engineering, University of Toronto, 2011.

[31] Goncalves, A.P.C., Fioravanti, A.R. \& Geromel, J.C., Filtering for Discrete-Time Markov Jump Systems with Network Transmitted Mode, $49^{\text {th }}$ IEEE Conference on Decision and Control, pp. 924-929, Dec. 15-17, 2010 . 